Fire Safety Review

The Performing Arts Center (PAC) Building 006 - The Christopher Cohan Center by

\title{
David Phillips
}

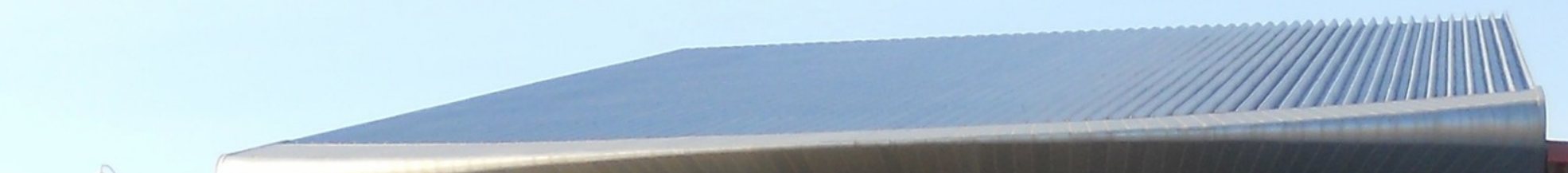

को

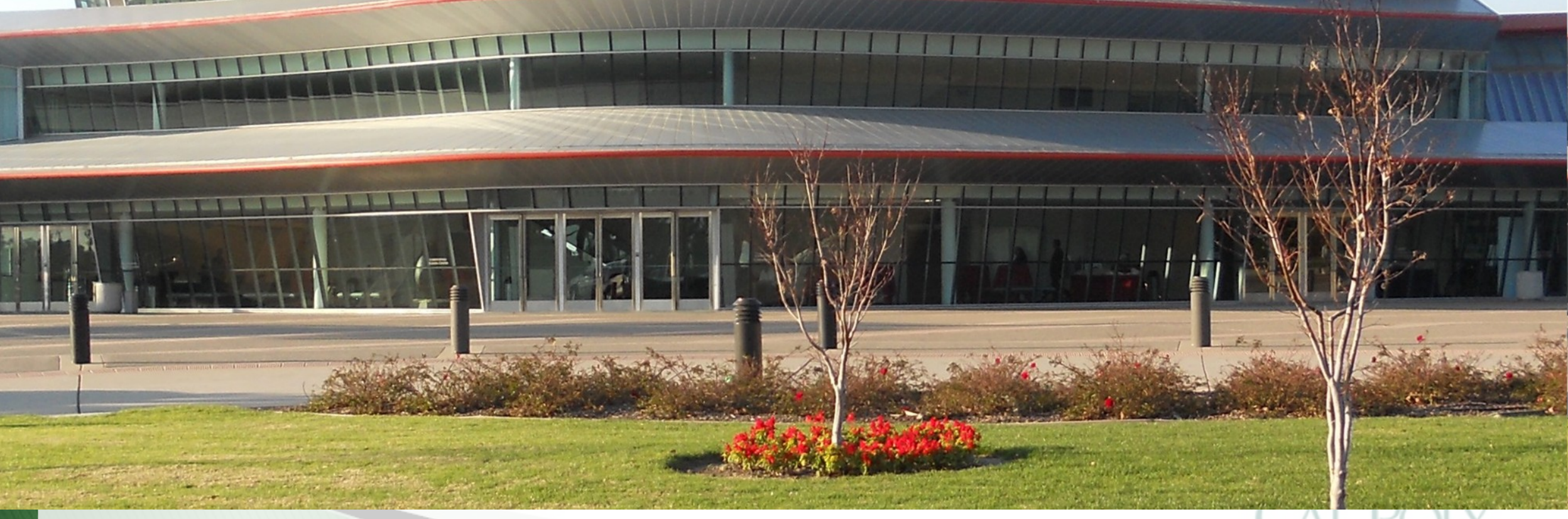

Image From: http://fasttimesaslo.files.wordpress.com/2011/01/dscn0510.jpg 


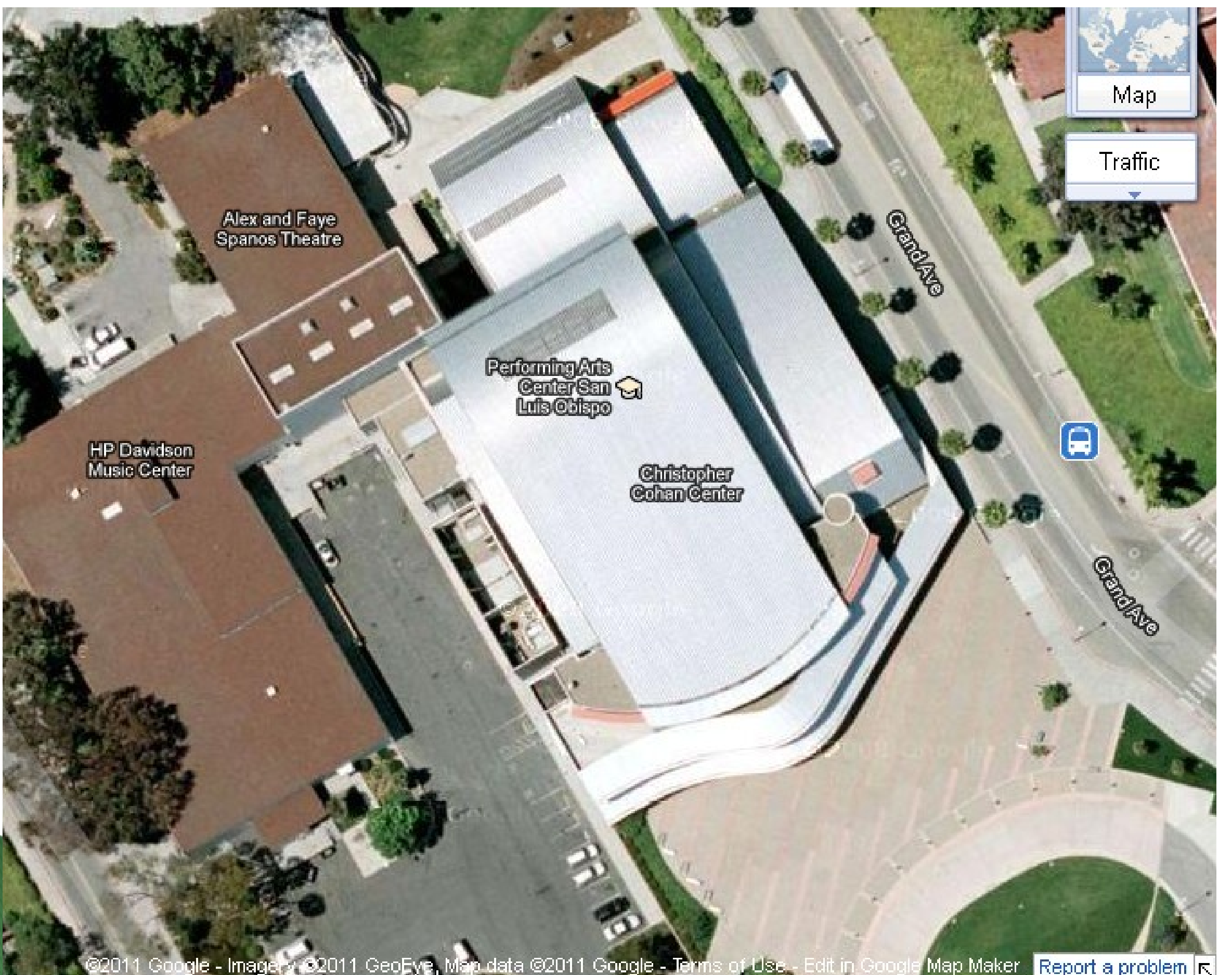




\section{Approach}

Prescriptive

- Floor plans, occupancy classifications, and exits

- Building photos

- Structural Protection

- Other protection features

- Hazards, unique features

\section{Performance}

- Design fire scenarios

- Analysis

- Results

- Conclusions 


\section{Occupancy Classifications}
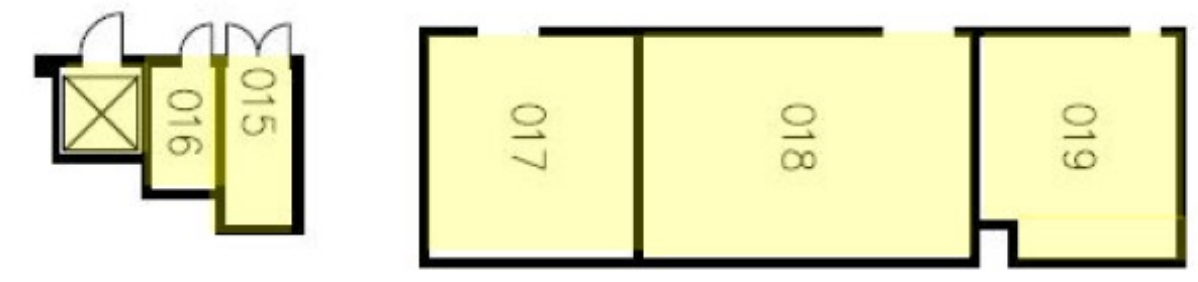

Equipment/Storage S

Assembly A-1

Assembly A-2

Assembly A-3

Business B

Kitchen
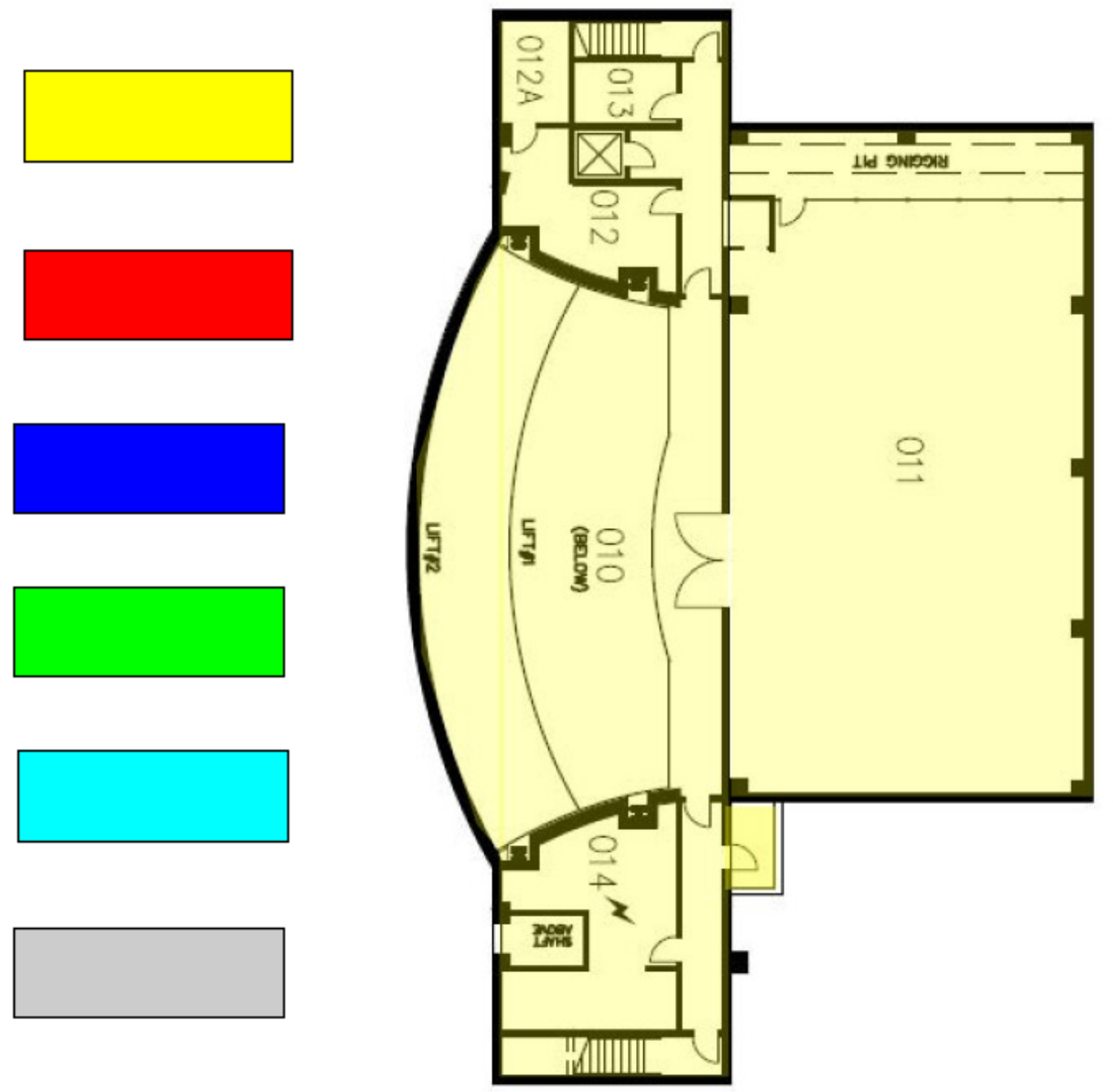


\section{Occupancy Classifications}

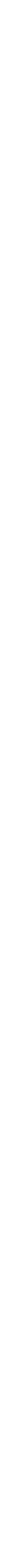




\section{Occupancv Classifications}

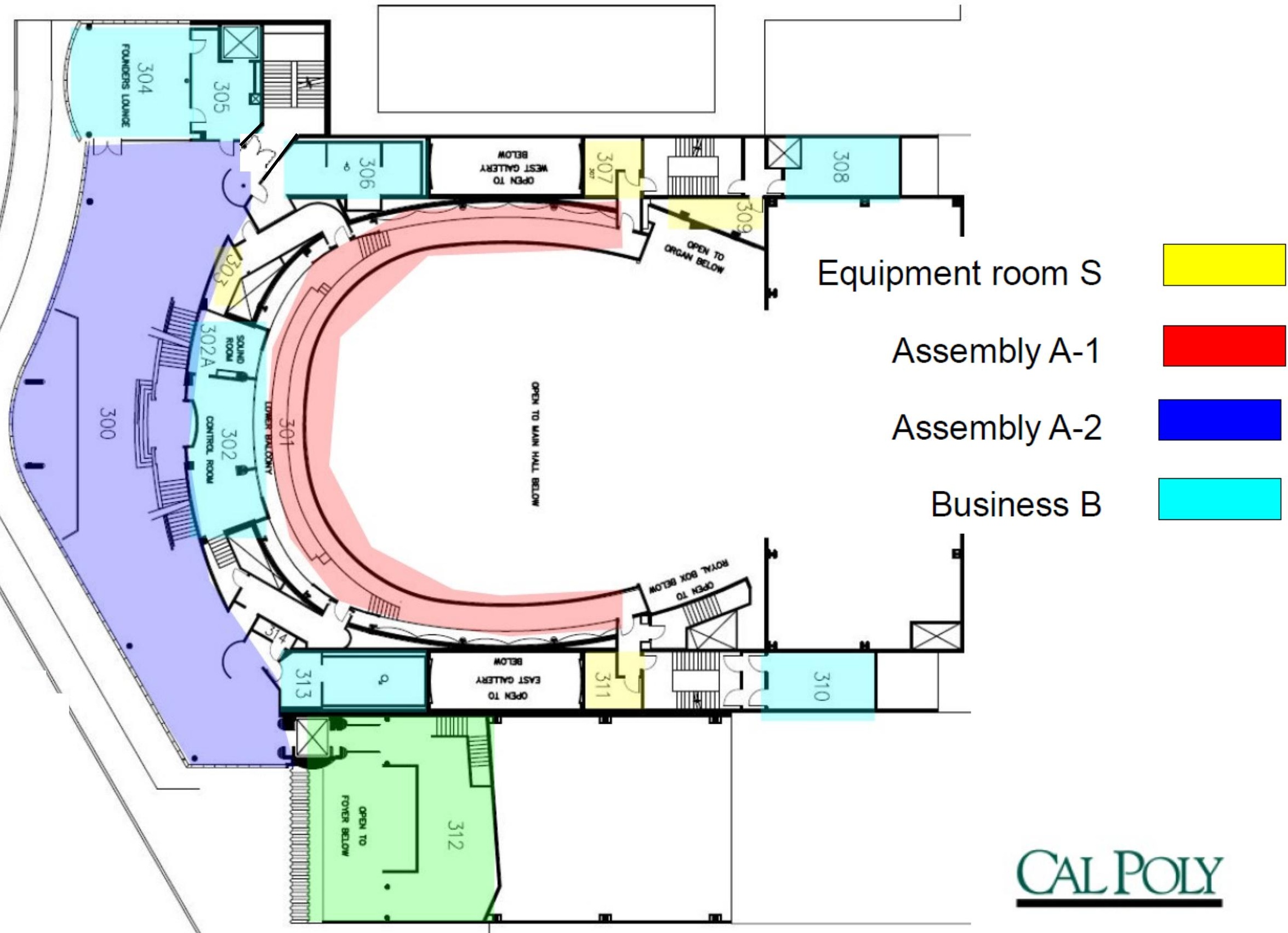




\section{Occupancy Classifications}

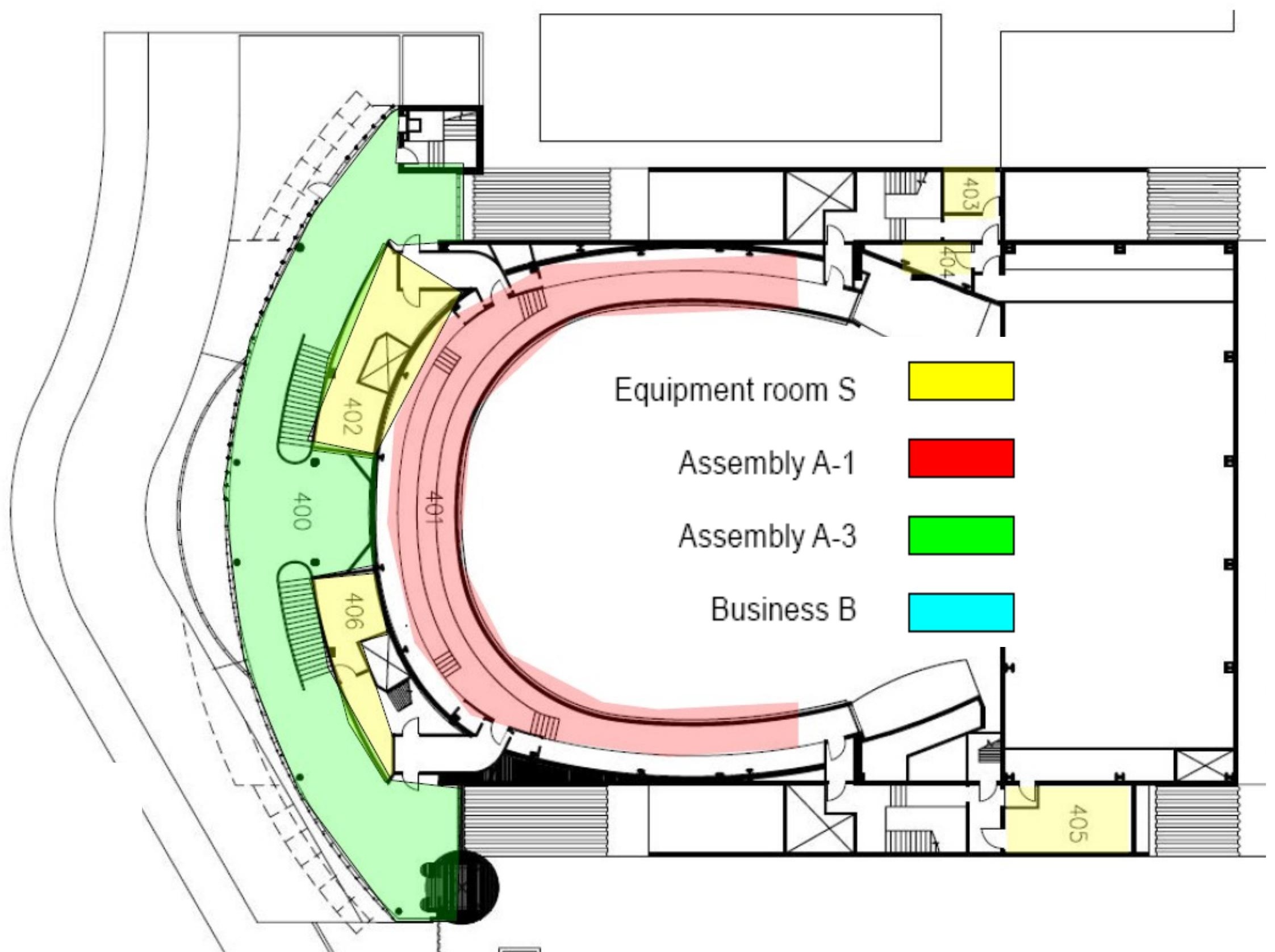




\section{Lower Level Exterior Exits}

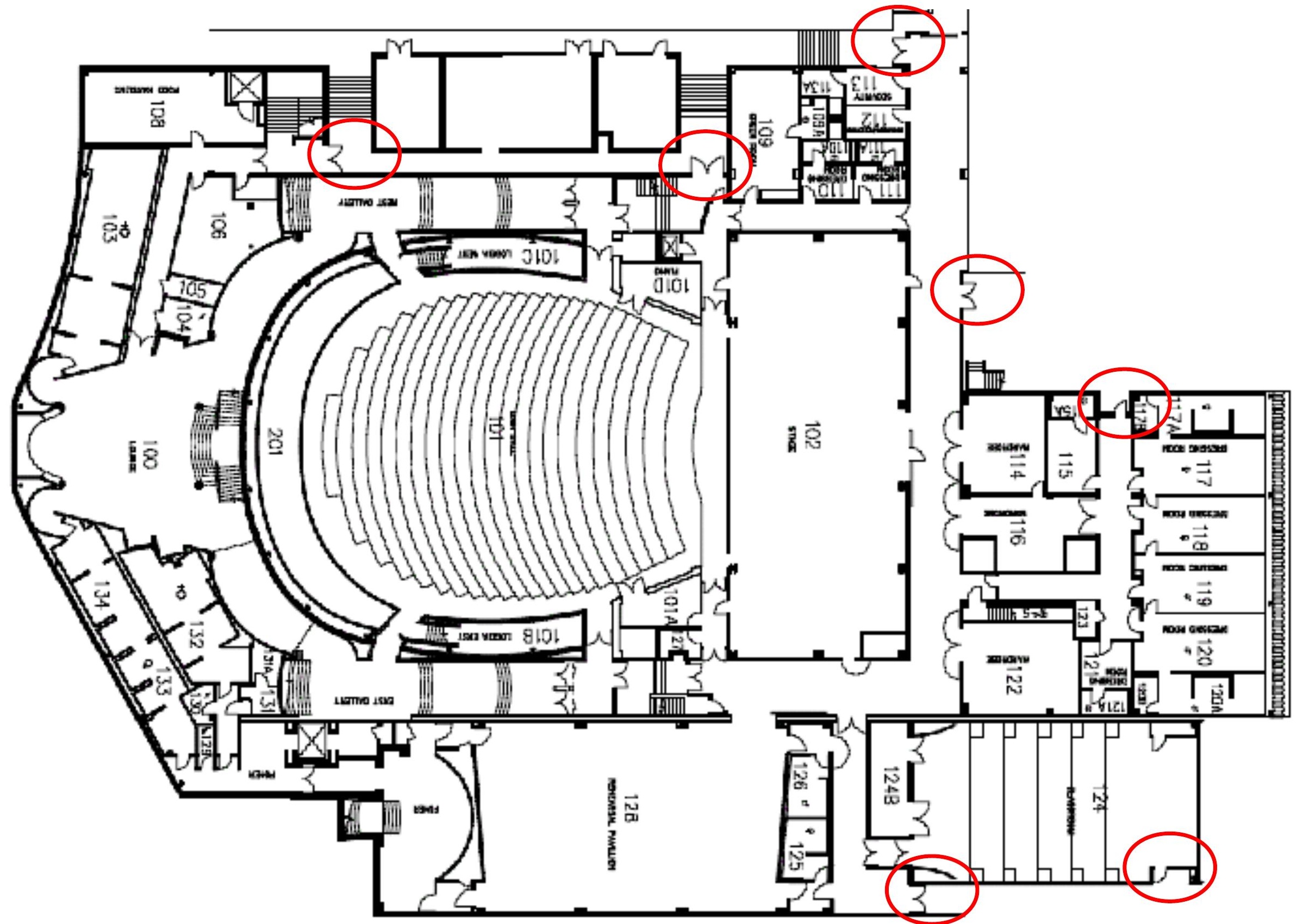




\section{Main Level Exits}
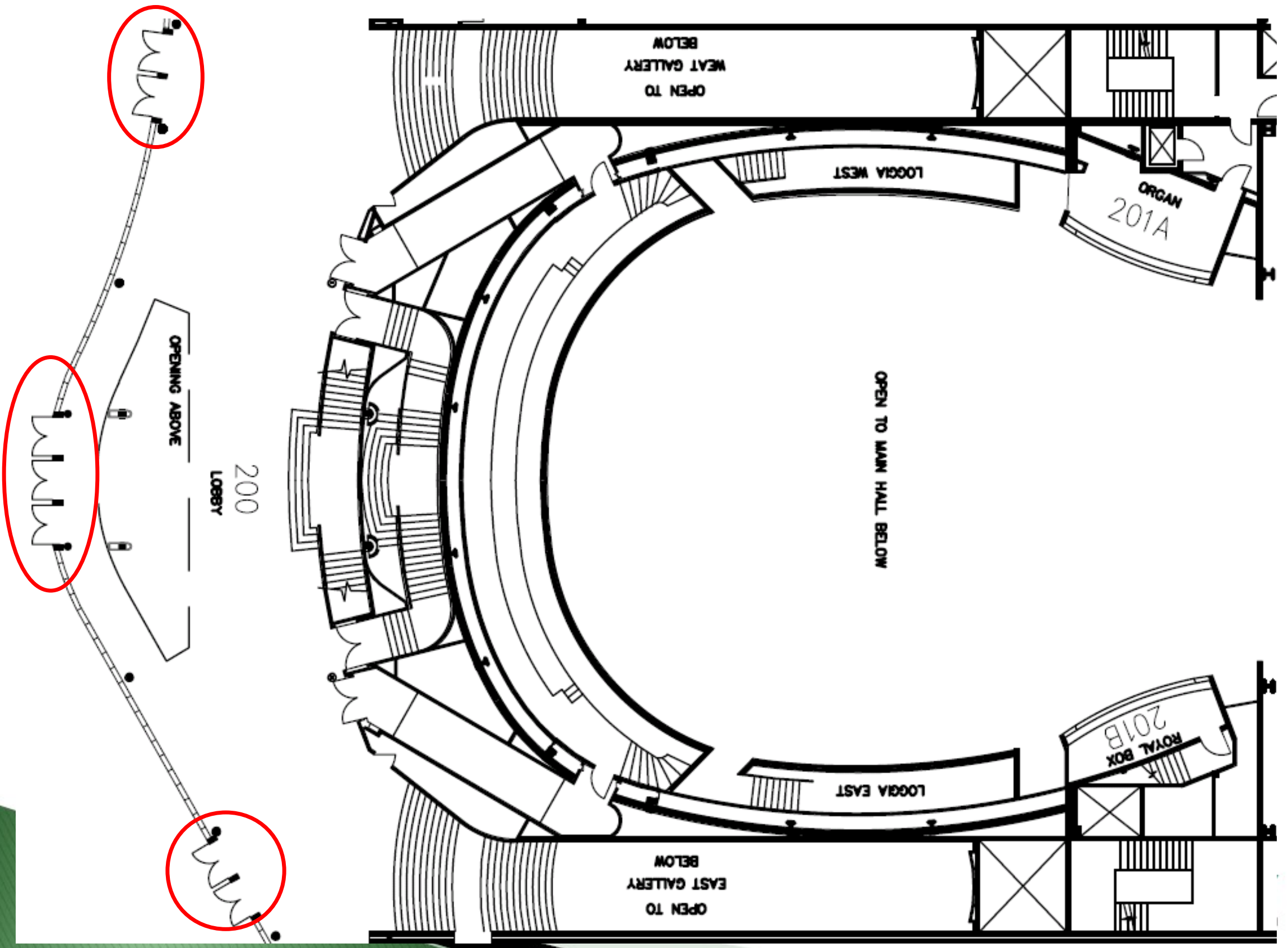


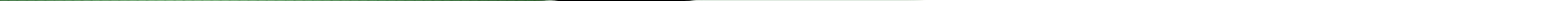




\section{Main Level Exits}

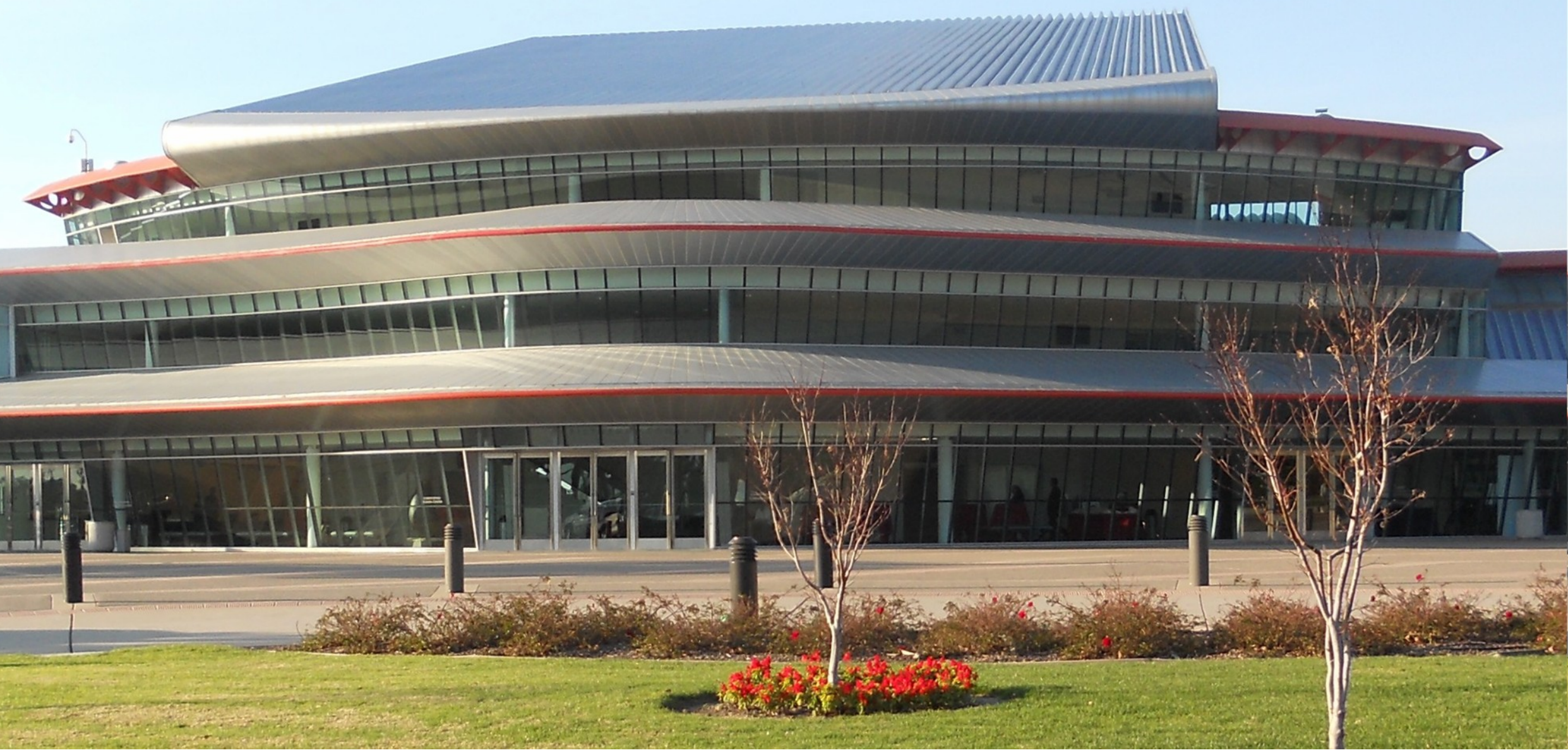

Image From: http://fasttimesaslo.files.wordpress.com/2011/01/dscn0510.jpg 


\section{Structural Protection}

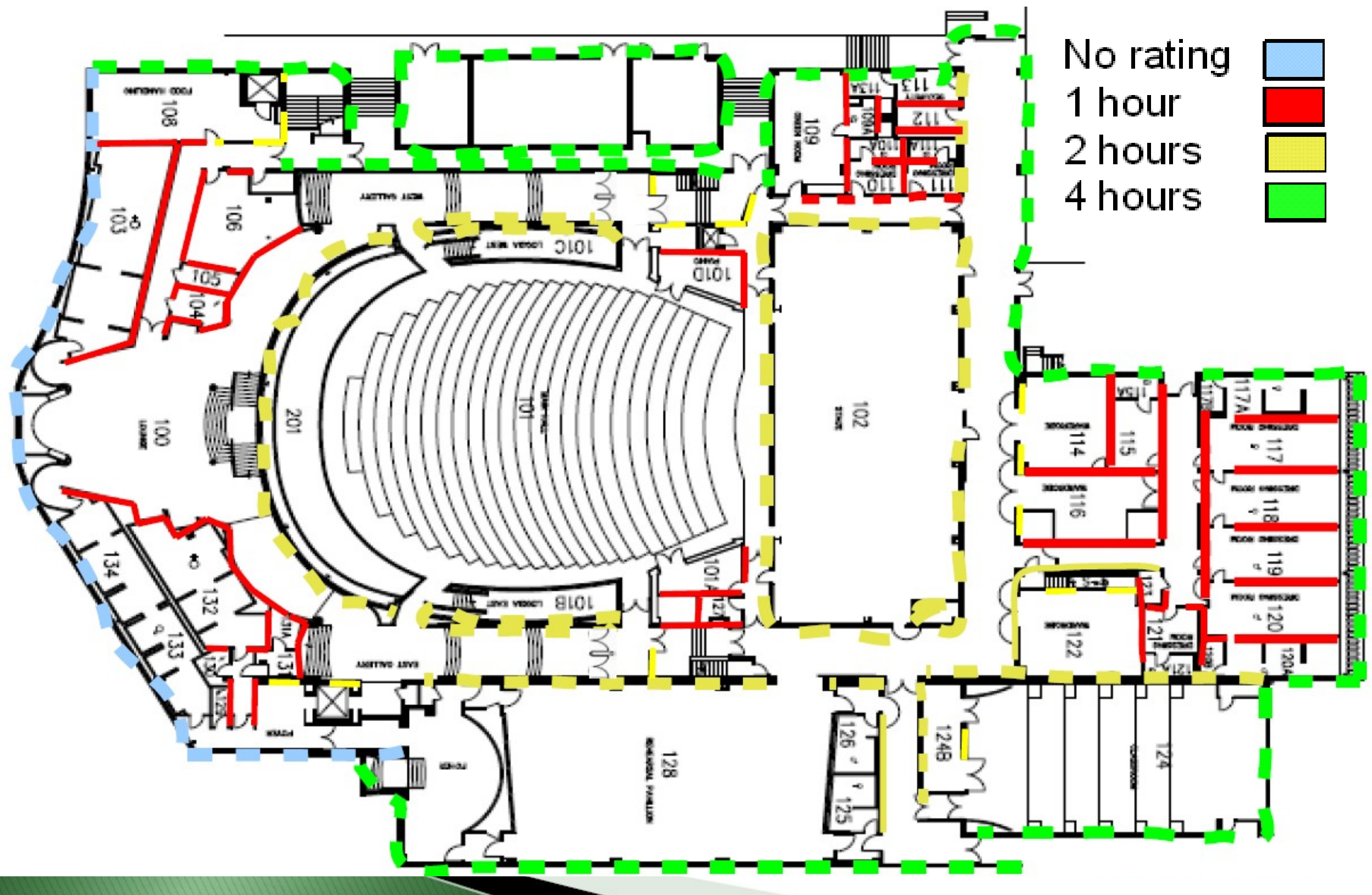




\section{Hall Exits}

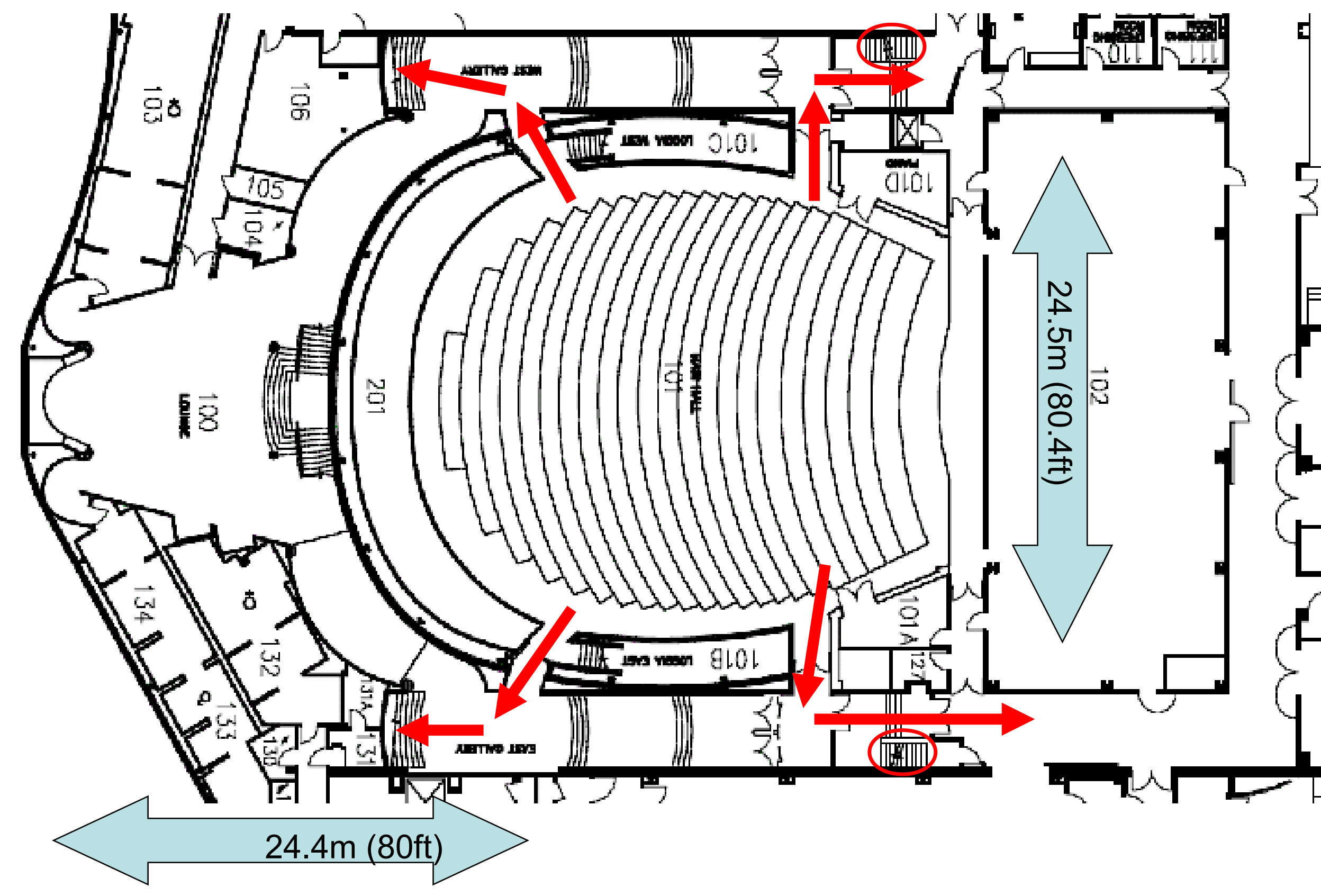




\section{Main Level Exits}

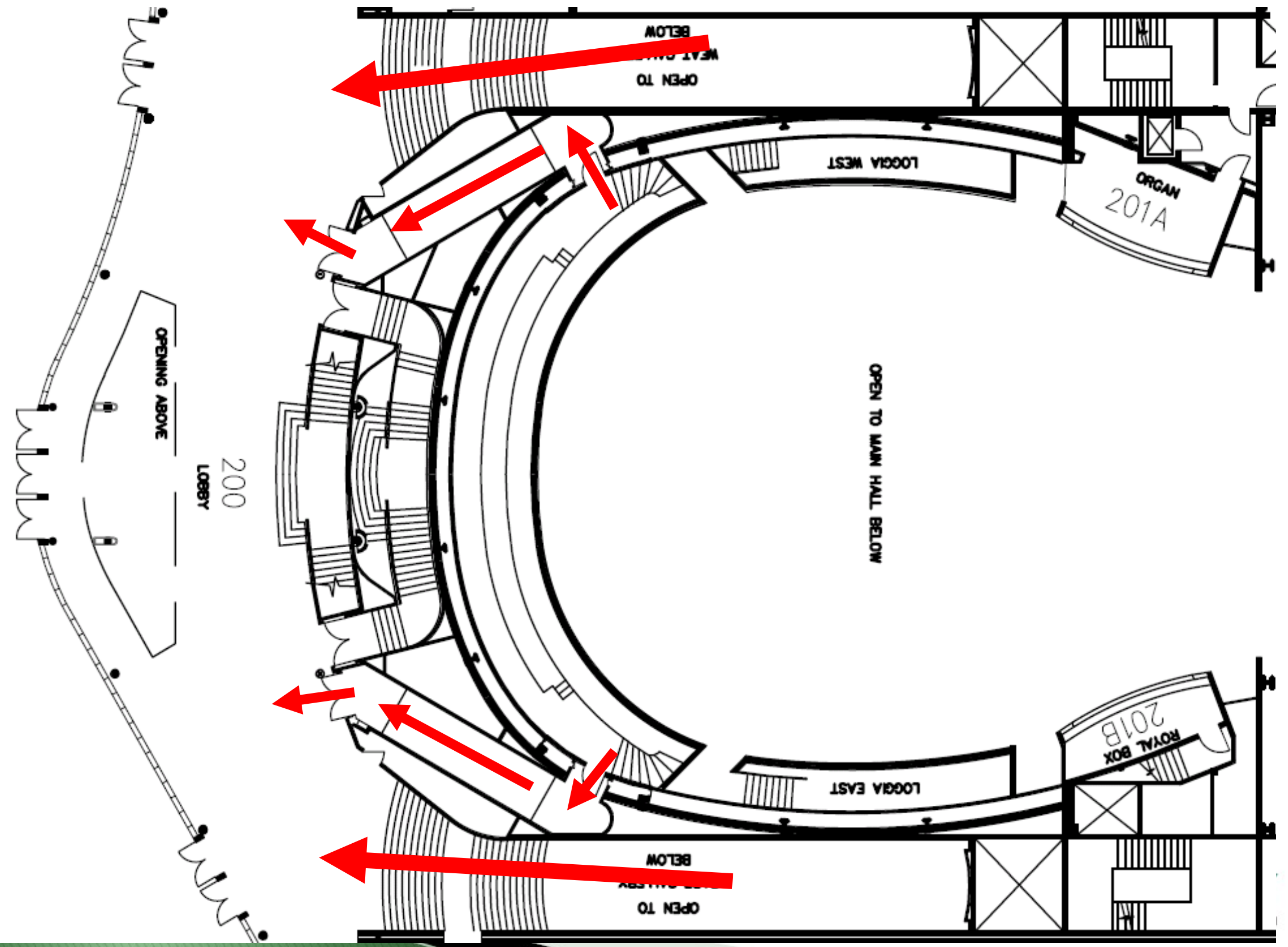




\section{Harman Hall}

\section{Max Capacity: 1282}

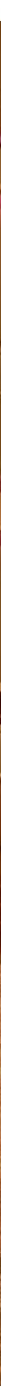

Image by: Malcolm Carlaw

http://www.flickr.com/photos/liahtsamples/5555005874/sizes/o/in/photostream/ 


\section{Main Floor: 808}

\section{Dress Circle: 153}

Balcony: 169

Gallery: 152

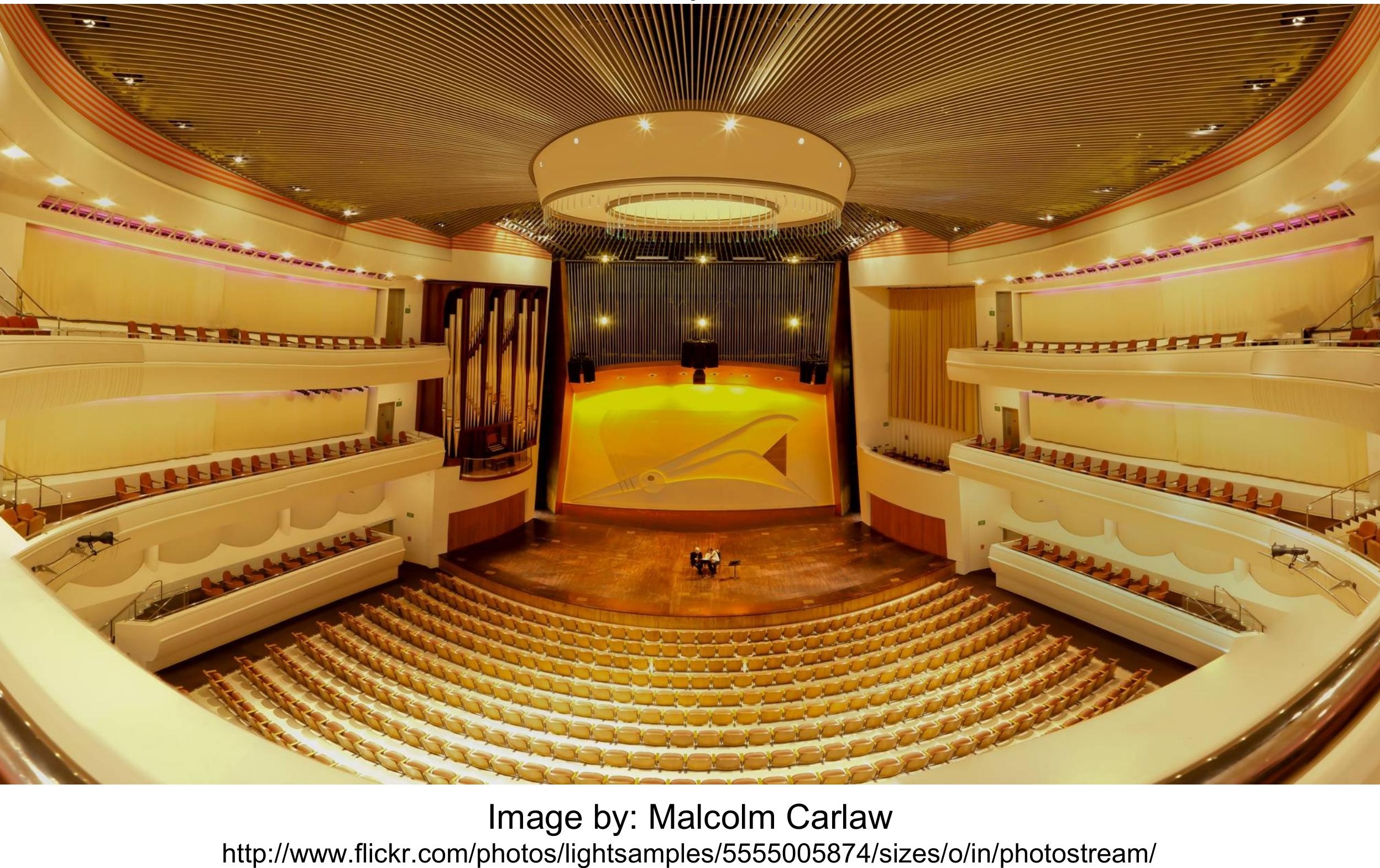




\section{Harman Hall}

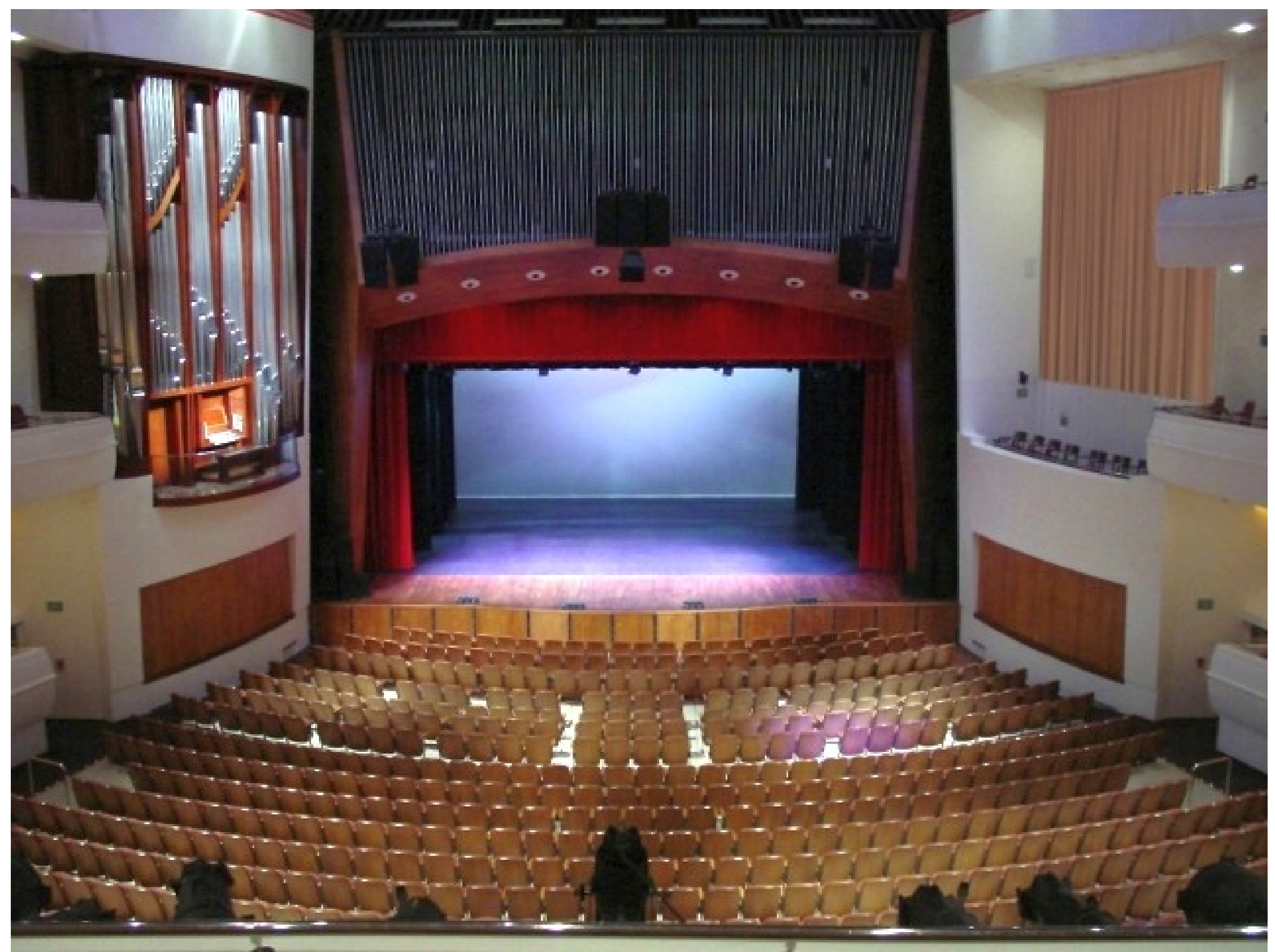

Image from: http://www.calpoly.edu/ ezabala/harmanhall.jpg 


\section{Other Required Features}

Fully Sprinklered, NFPA 13 Compliant

- B, A-1, and A-3 Occupancies - Light Hazard

- Storage, Kitchen, and Mechanical Rooms - Ordinary Hazard 1

- Stage and Orchestra Pit - Ordinary Hazard 2

-Gravity vents over stage - Fusible link and manual activation

-Fire curtain - Manual activation, rate of rise heat detector, and fusible link

-Voice occupant notification system

-Emergency power provided by a generator 


\section{Automatic Detection System}

NFPA 72 Compliant

\begin{tabular}{c|c|c|c|c|c|c|c}
\hline Device & $\begin{array}{c}\text { Speaker, } \\
\text { Strobe, } \\
\text { and } \\
\text { Voice }\end{array}$ & $\begin{array}{c}\text { Loading } \\
\text { Dock Bell }\end{array}$ & $\begin{array}{c}\text { Signal } \\
\text { Security / } \\
\text { Engineering }\end{array}$ & $\begin{array}{c}\text { Magnetic } \\
\text { Doors } \\
\text { Released }\end{array}$ & $\begin{array}{c}\text { Dampers } \\
\text { Close }\end{array}$ & $\begin{array}{c}\text { Elevator } \\
\text { Accordion } \\
\text { Doors }\end{array}$ & $\begin{array}{c}\text { Supervisory } \\
\text { Signal }\end{array}$ \\
\hline $\begin{array}{c}\text { Manual } \\
\text { Pull } \\
\text { Station }\end{array}$ & $\mathrm{X}$ & $\mathrm{X}$ & $\mathrm{X}$ & $\mathrm{X}$ & $\mathrm{X}$ & \\
\hline $\begin{array}{c}\text { Smoke } \\
\text { Detector }\end{array}$ & $\mathrm{X}$ & $\mathrm{X}$ & $\mathrm{X}$ & $\mathrm{X}$ & $\mathrm{X}$ & \\
\hline $\begin{array}{c}\text { Heat } \\
\text { Detector }\end{array}$ & $\mathrm{X}$ & & $\mathrm{X}$ & $\mathrm{X}$ & $\mathrm{X}$ & $\mathrm{X}$ & \\
\hline $\begin{array}{c}\text { Duct } \\
\text { Detector }\end{array}$ & $\mathrm{X}$ & & $\mathrm{X}$ & $\mathrm{X}$ & $\mathrm{X}$ & $\mathrm{X}$ & \\
\hline $\begin{array}{c}\text { Water } \\
\text { Flow } \\
\text { Alarm }\end{array}$ & $\mathrm{X}$ & $\mathrm{X}$ & & $\mathrm{X}$ & $\mathrm{X}$ & $\mathrm{X}$ & \\
\hline $\begin{array}{c}\text { Tamper } \\
\text { Switch }\end{array}$ & & & & & & & \\
\hline
\end{tabular}




\section{Hazards and Features}

Kitchen is equipped with an ANSUL range suppression system

Pipe organ is protected with a double interlock sprinkler system

Central station monitoring of an alarm panel annunciator

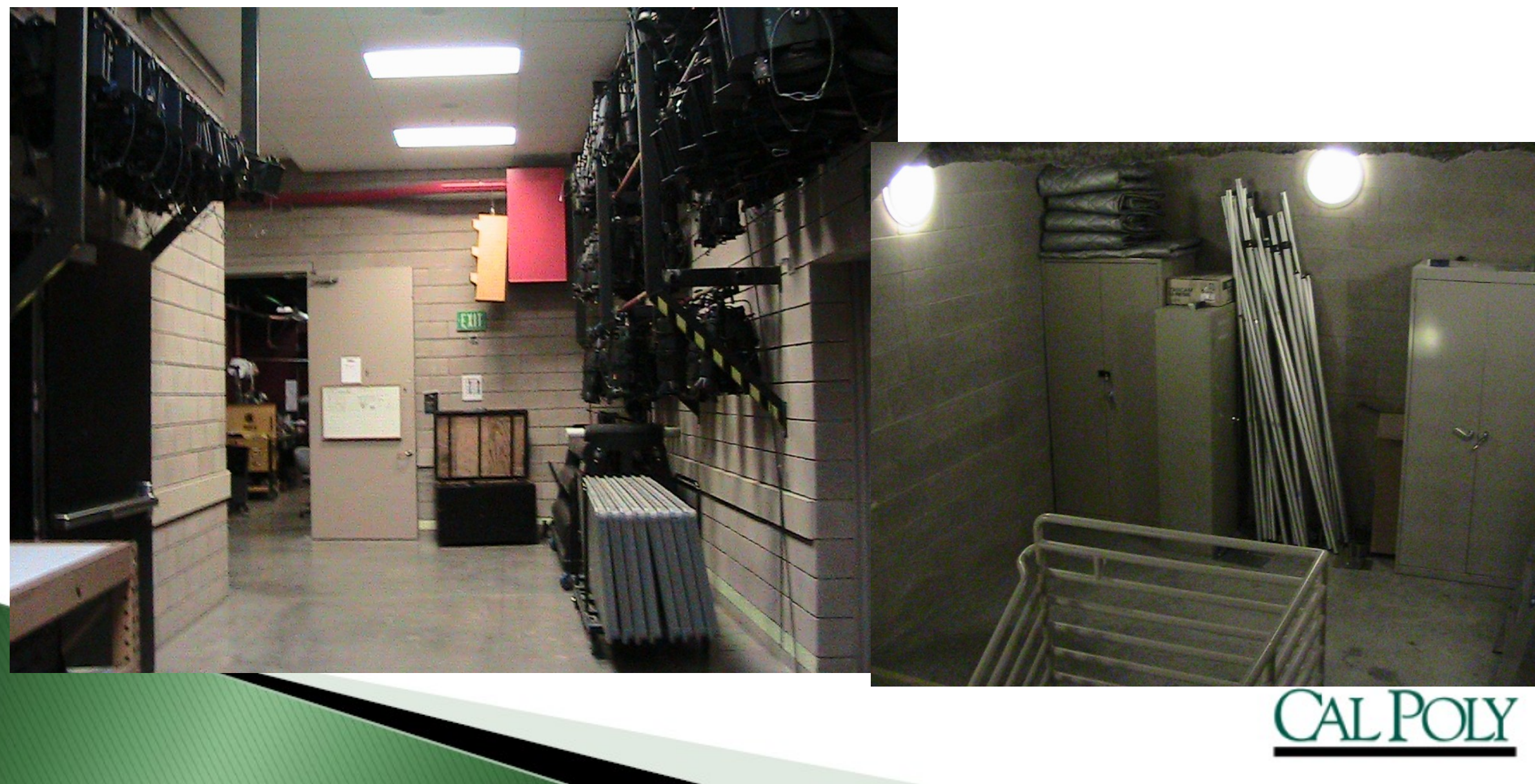




\section{Performance Based Analysis}

Scenarios selected from NFPA 101, the Life Safety Code

5.5.3.8 A fire originating in ordinary combustibles in a room or area with each passive or active fire protection system independently rendered ineffective.

- Assume fire curtain fails to deploy

- Fire growth modeled as a fast $\mathrm{t}^{2}$, NFPA Handbook Figure 3.7.2

- Heat of combustion, soot yield, and CO yield based on average of materials likely present for which such data is available

- Evacuation may not start immediately, because fog and smoke effects on stage may mask the presence of the early fire 


\section{Performance Based Analysis}

\section{Second Scenario}

5.5.3.2 An ultrafast-developing fire, in the primary means of egress, with interior doors open at the start of the fire.

- Fuel properties from the SFPE Handbook

- Assume 1 gallon of the liquid fuel, calculate burn out time based on heat of combustion and dictated HRR

- Assume fire spreads to seating, after which growth is a medium $\mathrm{t}^{2}$ fire, based on NFPA Handbook Figure 3.7.2

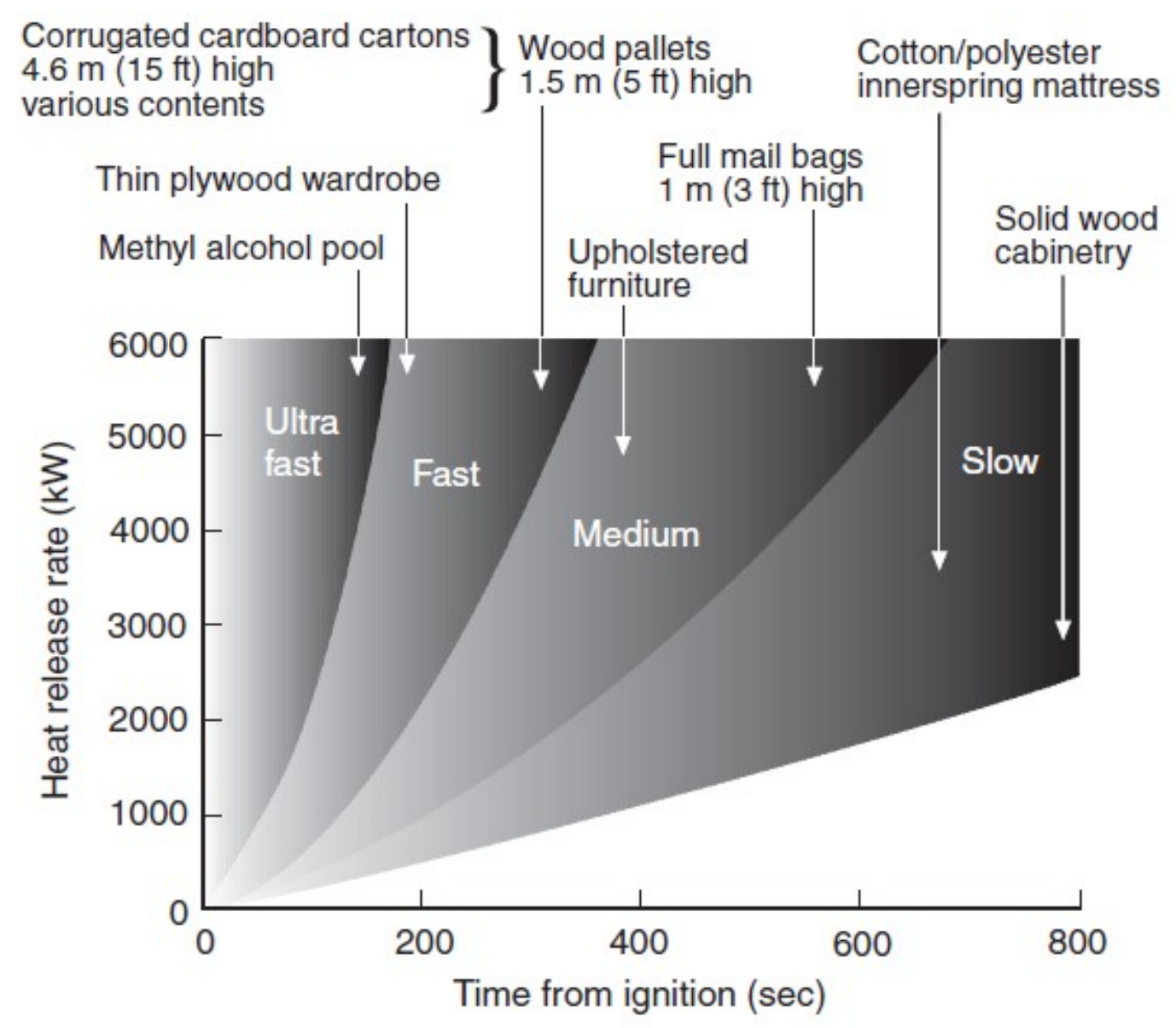

FIGURE 3.7.2 Set of $t$-Squared Growth Curves 


\section{Fuel Properties Scenario 1}

\section{Source: SFPE Handbook Table 3-4.14}

\begin{tabular}{|c|c|c|c|c|}
\hline Material & $\begin{array}{c}\text { Net heat of } \\
\text { combustion } \\
(\mathrm{kJ} / \mathrm{g})\end{array}$ & $\begin{array}{c}\text { Soot yield } \\
(\mathrm{g} / \mathrm{g})\end{array}$ & $\begin{array}{c}\text { CO yield } \\
(\mathrm{g} / \mathrm{g})\end{array}$ & $\begin{array}{c}\text { Radiant } \\
\text { Fraction }\end{array}$ \\
\hline Woods & & & & \\
\hline Red Oak & 12.4 & 0.015 & 0.004 & 0.371 \\
\hline Fir & 13.0 & & 0.004 & 0.377 \\
\hline Pine & 12.4 & & 0.005 & 0.298 \\
\hline Fiber board & 14.0 & & 0.015 & \\
\hline $\begin{array}{c}\text { Average of } \\
\text { known values }\end{array}$ & 12.9 & 0.015 & 0.007 & 0.349 \\
\hline
\end{tabular}




\section{Tenability Criteria}

Visibility: $10 \mathrm{~m}$ or greater

- $12 \mathrm{~m}$ maximum from any point on the balcony to an exit.

- $10 \mathrm{~m}$ visibility decreases chances of turn back behavior. (John L. Bryan, NFPA Handbook)

Temperature: Less then $60^{\circ} \mathrm{C}$.

- Limit at which 100\% humid air can be breathed. (David Purser, SFPE Handbook)

Toxicity: CO exposure less then $\mathbf{3 5 , 0 0 0 ~ p p m ~ * ~} \min$

- Exposures to $\mathrm{CO}$ greater then this can cause incapacitation. (Richard G. Gann, Nelson P. Bryner, NFPA Handbook)

Heat Flux: Less then $2.5 \mathrm{~kW} / \mathrm{m} 2$

- Severe skin pain in 1 second of exposure. (David Purser, SFPE Handbook) 


\section{RSET}

Evacuation time determined by Pathfinder and compared with SFPE method hand calculations.

Main Seating - Two 72 inch doors and two 44 inch doors available

-SFPE method, spreadsheet- 2.5 minutes

- Pathfinder steering mode -2.0 minutes

- Pathfinder SFPE mode, collisions enabled - 2.6 minutes

Main Seating - One 72 inch door unavailable

- Pathfinder SFPE mode, collisions enabled - $\mathbf{3 . 9}$ minutes

Gallery Seating - Four 36 inch doors available

- Pathfinder SFPE mode, collisions enabled - 1.4 minutes

Gallery Seating - Three 36 inch doors available

- Pathfinder SFPE mode, collisions enabled - $\mathbf{1 . 5}$ minutes 


\section{Pathfinder}

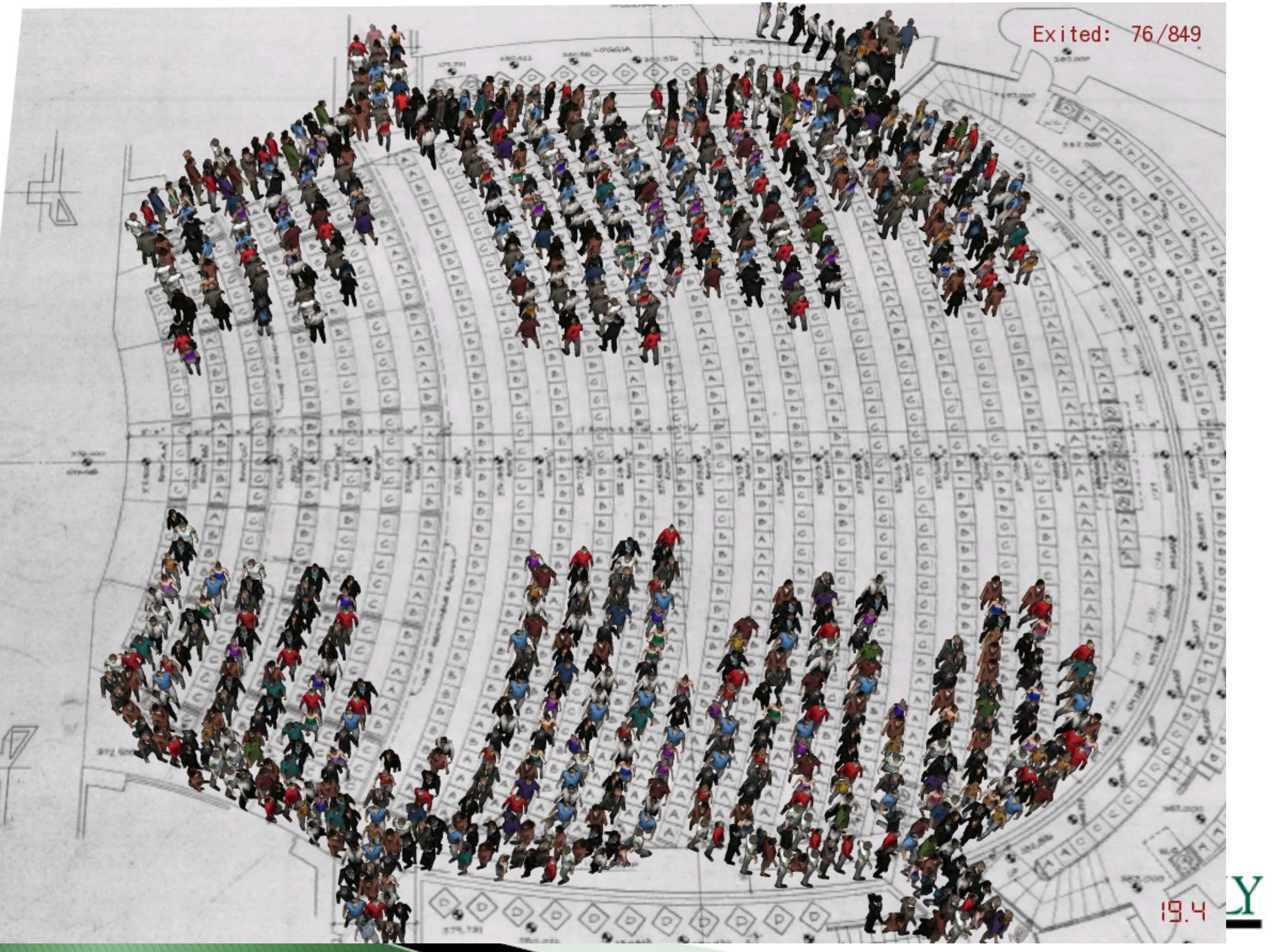




\section{Scenario One Results}

\section{Tenability using CFAST}

ASET for $10 \mathrm{~m}$ visibility criteria is about 4 minutes for the gallery level, using the SFPE mass optical density method.

Smoke layer has descended to $6 \mathrm{ft}$ above gallery seating in 3.8 minutes.

\section{Sign Visibility}

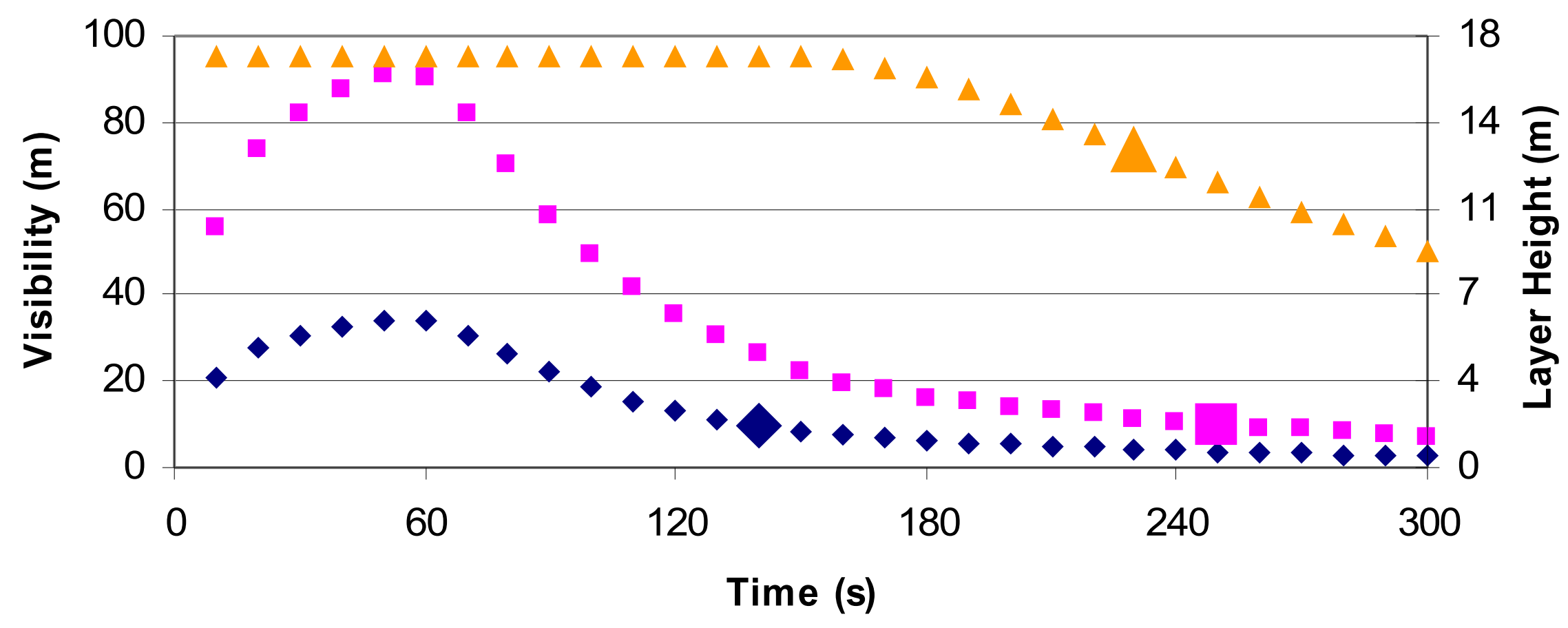

- Reflective Sign - Emitting Sign $\triangle$ Layer Height 


\section{Scenario One Results}

Tenability using FDS

ASET for $10 \mathrm{~m}$ visibility criteria is not reached for the illuminated sign over the gallery seating in 15 minutes.

Smoke layer has descended to $6 \mathrm{ft}$ above gallery seating in 6.8 minutes.

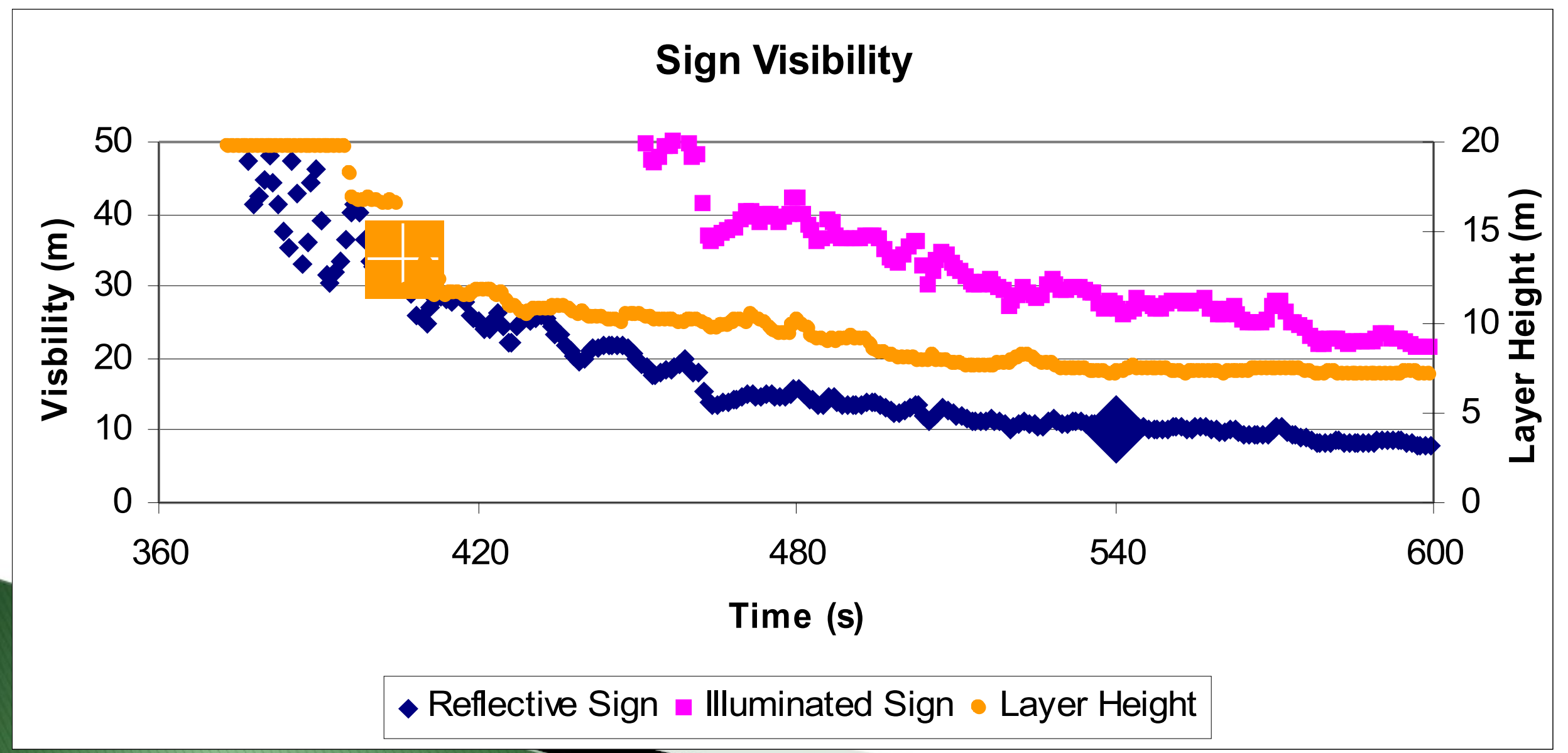




\section{Scenario One Results}

Tenability using CFAST

At 2.5 minutes, the upper layer in the CFAST model had just lowered to the top of the proscenium arch, so no visibility or CO tenability criteria would have been met.

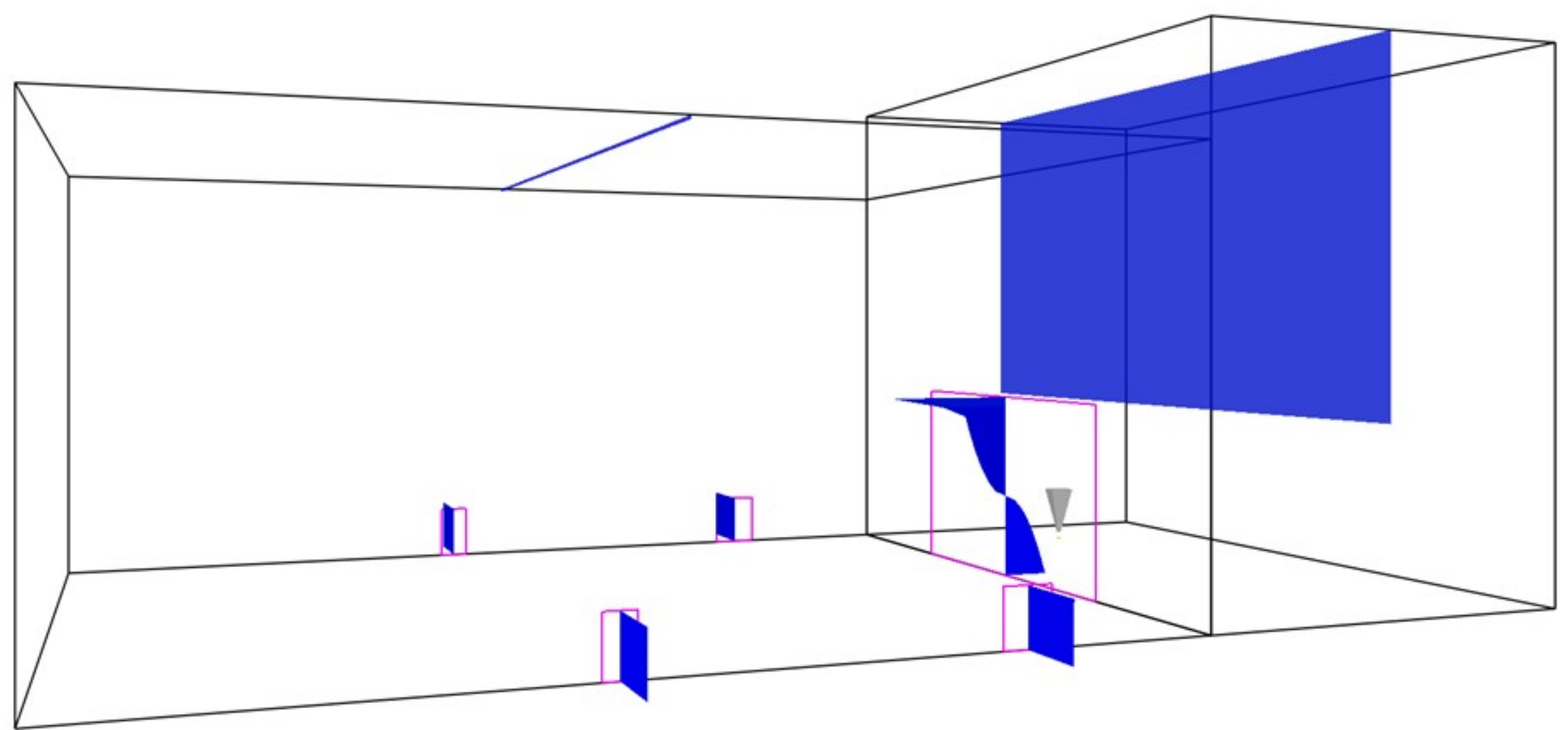


Tenability using FDS

At 4 minutes, smoke is just beginning to escape beneath the proscenium wall.

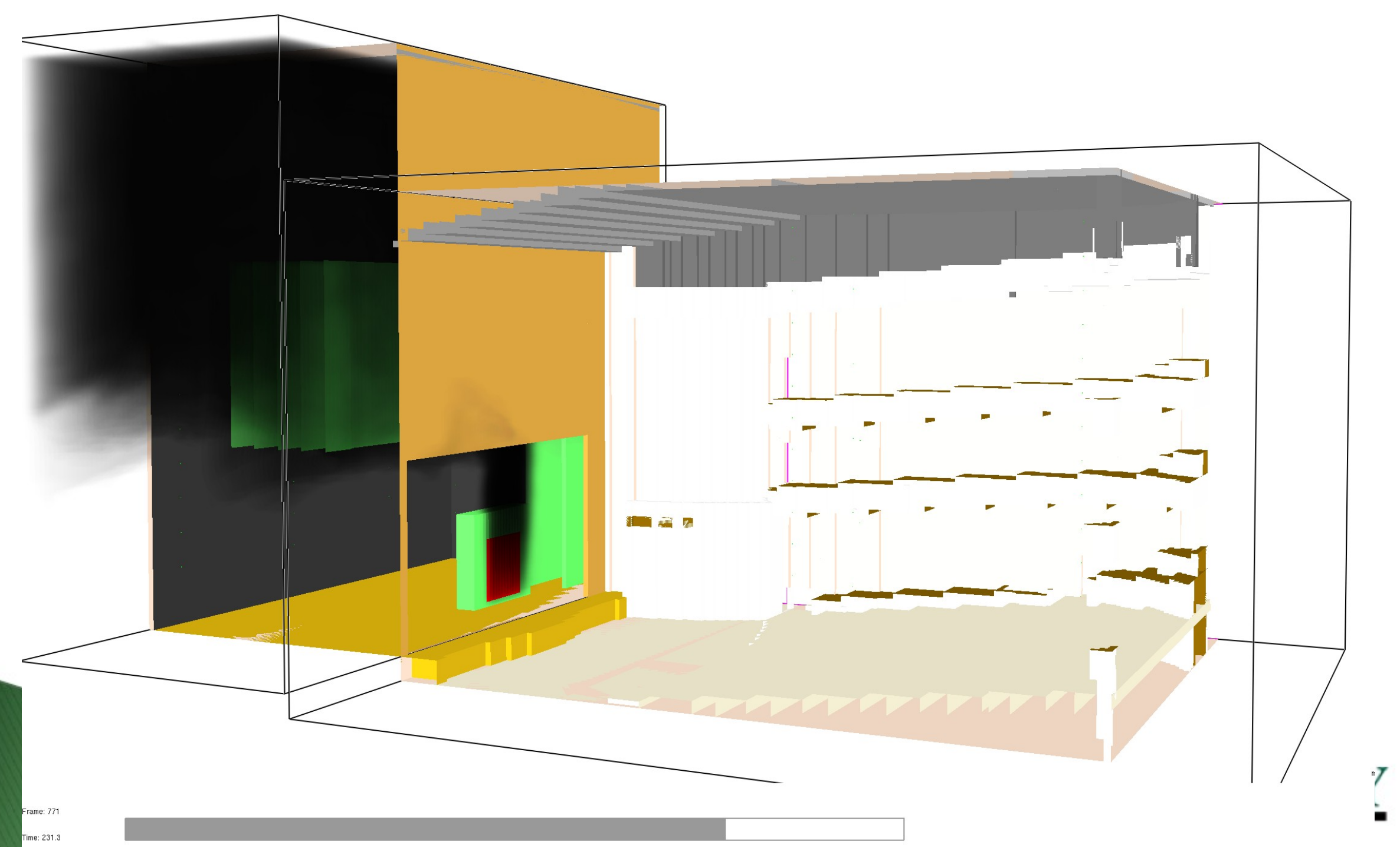


ASET for $10 \mathrm{~m}$ visibility criteria is about 3.2 minutes for the gallery level, using the SFPE mass optical density method.

Smoke layer has descended to $6 \mathrm{ft}$ above gallery seating in 1.2 minutes.

\section{Sign Visibility}

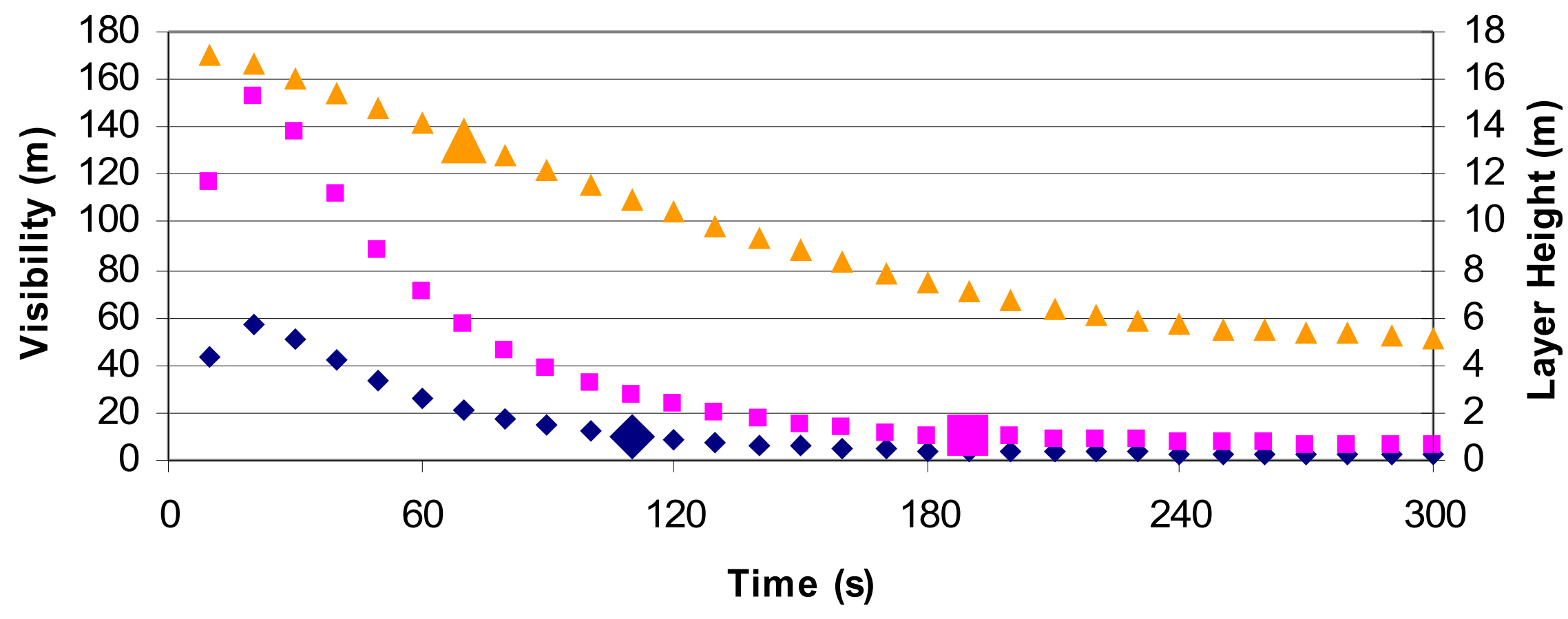

- Reflective Sign $=$ Emitting Sign $\Delta$ Layer Height 


\section{Scenario Two Results}

Tenability using FDS

ASET for $10 \mathrm{~m}$ visibility criteria is reached for the illuminated sign over the gallery seating in 7.6 minutes.

Smoke layer has descended to $6 \mathrm{ft}$ above gallery seating in 2.2 minutes.

\section{Sign Visibility}

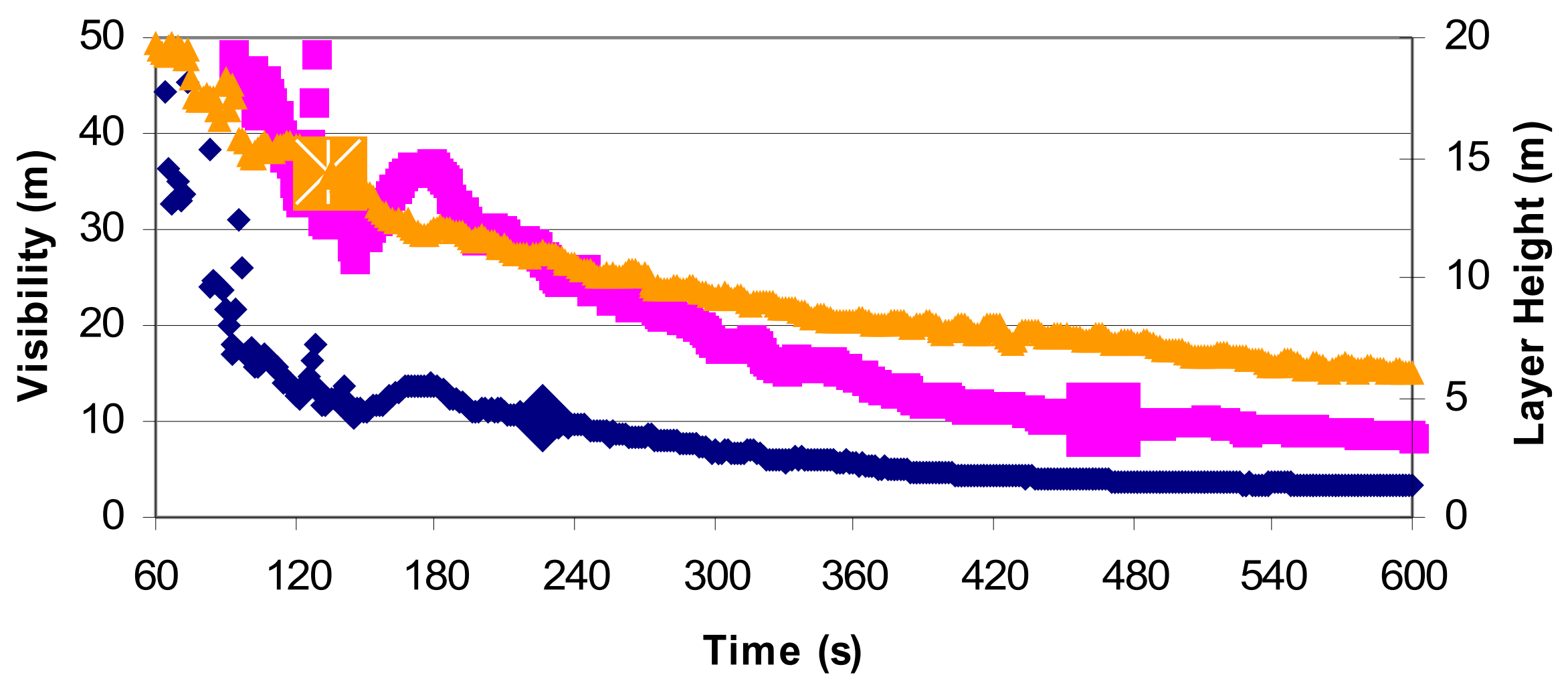

- Reflective Sign $\square$ Illuminated Sign $\Delta$ Layer Height 


\section{Scenario Two}

CFAST vs. FDS

Transport lag about 22 seconds

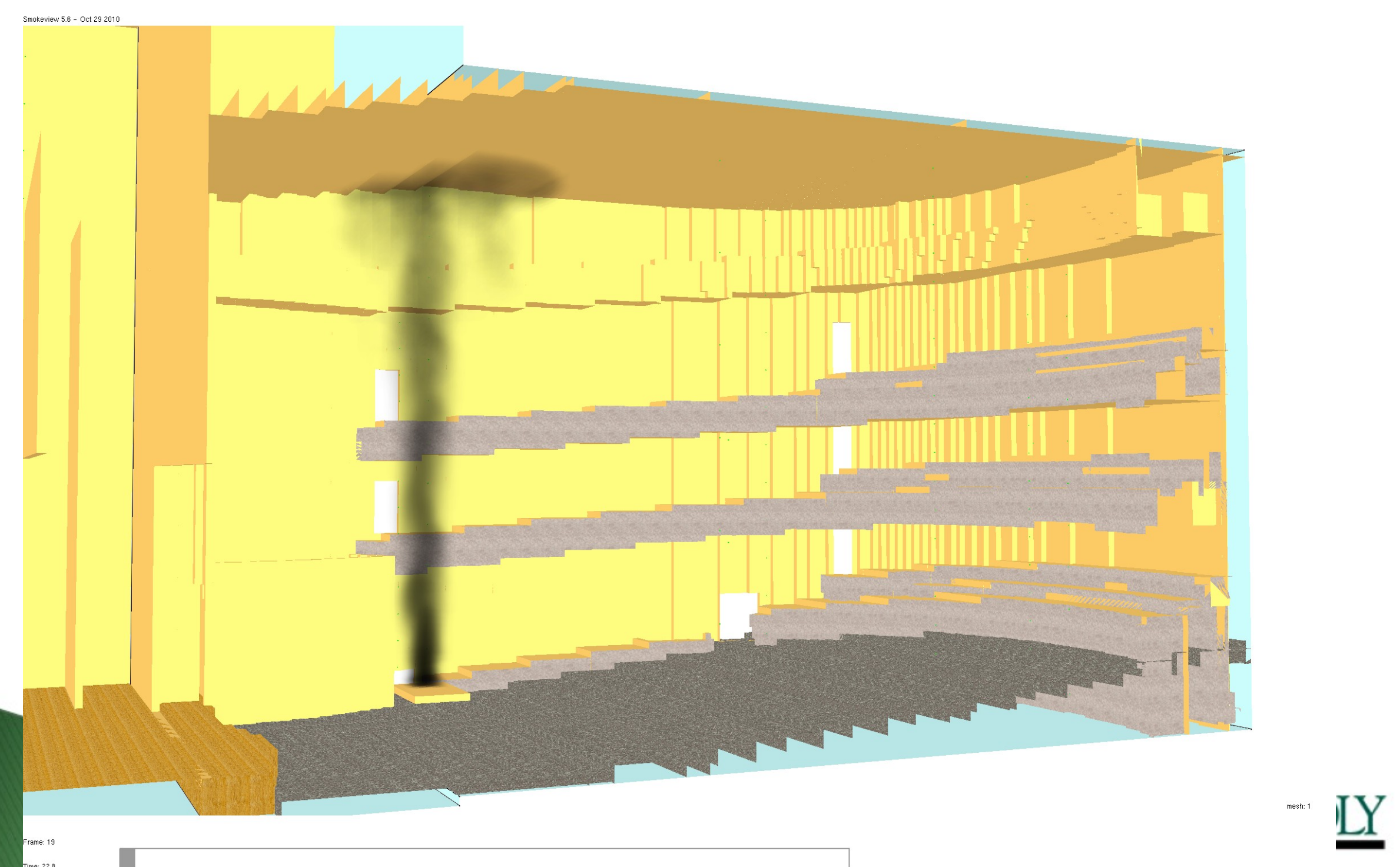




\section{Scenario Two \\ CFAST vs. FDS}

70 seconds

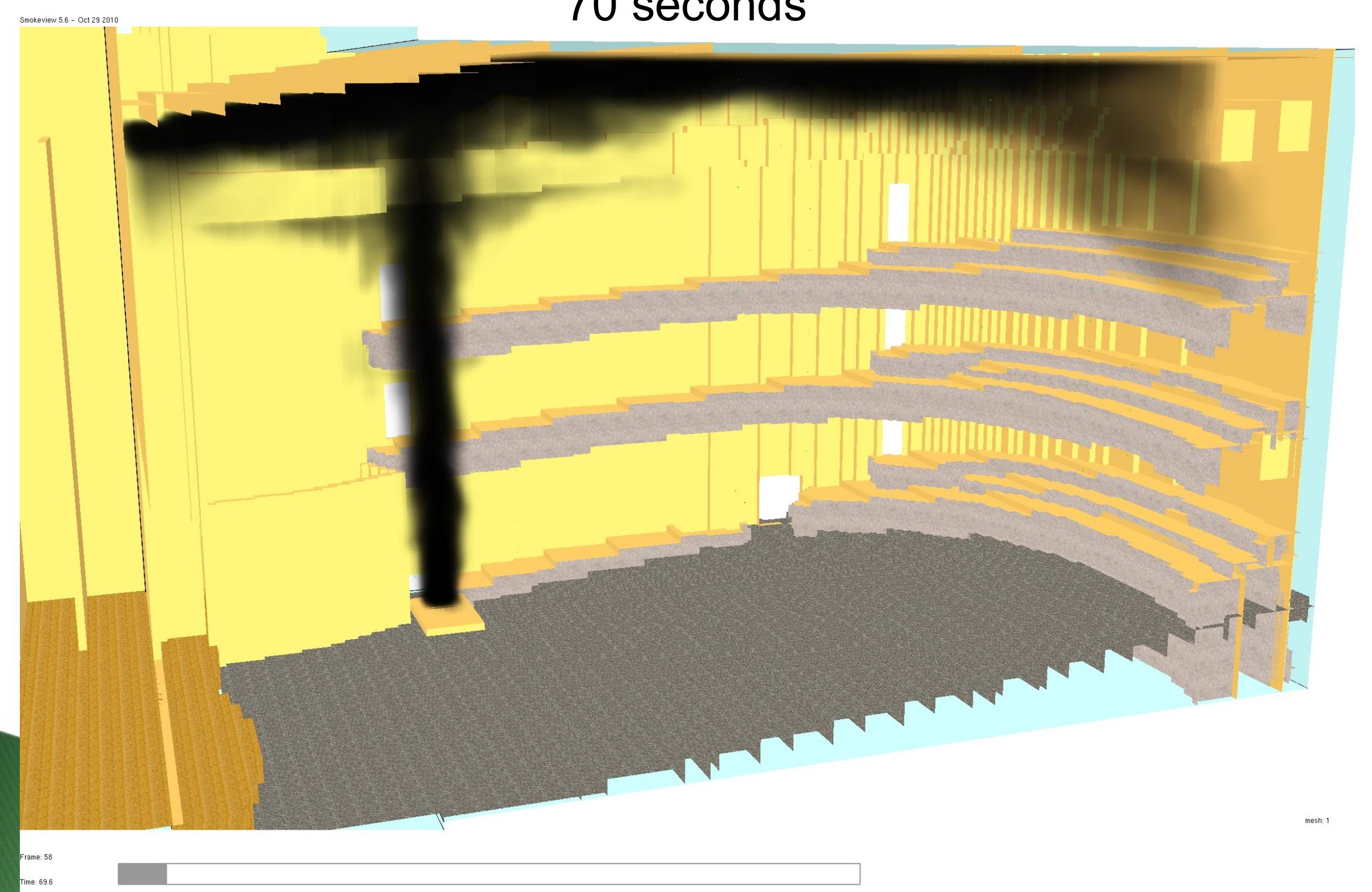




\section{Scenario Two}

CFAST vs. FDS

130 seconds 


\section{Results}

\begin{tabular}{|c|c|c|c|c|c|}
\hline \multicolumn{7}{|c|}{ Scenario 1 } \\
\hline $\begin{array}{c}\text { Criteria and } \\
\text { Location }\end{array}$ & $\begin{array}{c}\text { RSET } \\
\text { (minutes) }\end{array}$ & $\begin{array}{c}\text { CFAST } \\
\text { ASET } \\
\text { (minutes) }\end{array}$ & $\begin{array}{c}\text { FDS } \\
\text { ASET } \\
\text { (minutes) }\end{array}$ & $\begin{array}{c}\text { Margin } \\
\text { CFAST } \\
\text { (minutes) }\end{array}$ & $\begin{array}{c}\text { Margin } \\
\text { FDS } \\
\text { (minutes) }\end{array}$ \\
\hline $\begin{array}{c}\text { Layer } \\
\text { Gallery }\end{array}$ & 1.4 & 3.8 & 6.8 & 2.4 & 5.4 \\
\hline $\begin{array}{c}\text { Visibility } \\
\text { Gallery }\end{array}$ & 1.4 & 4 & $>15$ & 2.6 & $>13.6$ \\
\hline $\begin{array}{c}\text { Visibility } \\
\text { Floor }\end{array}$ & 2.6 & 7 & $>15$ & 4.4 & $>12.4$ \\
\hline $\begin{array}{c}\text { Temp } \\
\text { Gallery }\end{array}$ & 1.4 & 9 & $>15$ & 7.6 & $>13.6$ \\
\hline Temp Floor & 2.6 & 9 & $>15$ & 6.4 & $>12.4$ \\
\hline
\end{tabular}




\section{Results}

\section{Scenario 2}

\begin{tabular}{|c|c|c|c|c|c|}
\hline $\begin{array}{c}\text { Criteria and } \\
\text { Location }\end{array}$ & $\begin{array}{c}\text { RSET } \\
\text { (minutes) }\end{array}$ & $\begin{array}{c}\text { CFAST } \\
\text { ASET } \\
\text { (minutes) }\end{array}$ & $\begin{array}{c}\text { FDS } \\
\text { ASET } \\
\text { (minutes) }\end{array}$ & $\begin{array}{c}\text { Margin } \\
\text { CFAST } \\
\text { (minutes) }\end{array}$ & $\begin{array}{c}\text { Margin } \\
\text { FDS } \\
\text { (minutes) }\end{array}$ \\
\hline Layer Gallery & 1.5 & 1.2 & 2.2 & -0.3 & 0.7 \\
\hline $\begin{array}{c}\text { Visibility } \\
\text { Gallery }\end{array}$ & 1.5 & 3.2 & 3.7 & 1.7 & 2.2 \\
\hline Visibility Floor & 3.9 & 7 & $>17$ & 3.1 & $>13.1$ \\
\hline Temp Gallery & 1.5 & 4 & 11.1 & 2.5 & 9.6 \\
\hline Temp Floor & 3.9 & 7.3 & $>17$ & 3.4 & $>13.1$ \\
\hline
\end{tabular}




\section{Summary}

\section{Prescriptive}

- Building compliancy with investigated code isssues

\section{Performance}

- Scenario 1

- Greater then 2.5 minutes for detection and pre-movement

\section{- Scenario 2}

- Little to no margin for detection and pre-movement before smoke layer reaches occupants

- Small margin between visibility criteria and evacuation

\section{Models}

- CFAST model neglects transport lag and has greater entrainment when compared to the FDS model 